\title{
NUEVO REGISTRO DE PSEUDOCIDARIS SPP. (ECHINOIDEA: HEMICIDARIDAE) DEL CRETÁCICO INFERIOR (VALANGINIANO-HAUTERIVIANO) DE ZAPATOCA (SANTANDER, COLOMBIA)
}

\author{
(New record of Pseudocidaris sp. (Echinoidea:Hemicidaridae) of lower Cretaceous \\ (Valanginian-Hauterivian) from Zapatoca (Santander, Colombia)
}

\author{
Javier H. Jerez-Jaimes, Álvaro Sergio Osma Duarte y Joan Sebastián Puentes Pérez*
}

*Escuela de Geología, Universidad Industrial de Santander. javjerez@gmail.com, jaominosma@gmail.com, joandeca@ hotmail.es

(Recibido enero 7 de 2015 y aceptado marzo 2 de 2015)

\section{Resumen:}

En este estudio se reporta y describe un equinodermo regular del género Pseudocidaris en la Formación Rosablanca del Cretácico Inferior en el municipio de Zapatoca (Santander, Colombia). La especie presenta poca sinuosidad en los ambulacros y un disco apical dicíclico. Un análisis filogenético exploratorio muestra que el linaje de la especie se originó en el Calloviano con $P$. romani y que está relacionada con $P$. lusitánica (Oxfordiano) y P. clunifera (Barremiano-Aptiano)

Palabras clave: erizo de mar, erizo regular, Formación Rosablanca, fósil.

\section{INTRODUCCIÓN}

En el área del municipio de Zapatoca aflora la Formación Rosablanca (Cretácico: Valanginiano-Hauteriviano inferior), cuyo intervalo superior está compuesto en su mayor parte por limolitas arcillosas interestratificadas con micritas y biomicritas, donde abundan los Thalassinoides (Ward, Goldsmith, Cruz \& Jaramillo, 1973). El registro de la fauna de equinodermos fósiles en dicha formación se reduce a las especies Toxaster colombianus y Diplopodia spp (Etayo-Serna, 1964; Guzmán, 1985). Patarroyo (2002) en su descripción de equinoideos del miembro calcáreo superior (Cretácico: Hauteriviano

\section{Abstract:}

In this study it is reported and described a regular echinoderm of Pseudocidaris genus in the Rosablanca Formation of Lower Cretaceous at the municipality of Zapatoca (Santander, Colombia). The species shows little sinuosity in the ambulacra and a dyciclic apical disc. An exploratory phylogenetic analysis showed that the lineage of the Zapatoca species was originated in the Callovian with $P$. Romani and that it is related with $P$. lusitanica (Oxfordian) and P. clunifera ( Barremian-Aptian)

Keywords: fossil, Rosablanca Formation, regular urchin, sea urchin.

tardío-Barremiano temprano) de la Formación Tibasosa en Boyacá describe a Pseudocidaris cf. clunifera, de igual forma presenta una lista de publicaciones relacionadas con equinodermos del país. En este trabajo se describe y determina una especie de equinodermo regular relacionado con el género Pseudocidaris.

\section{MATERIALES Y MÉTODOS}

Los especímenes fueron colectados durante las visitas de campo del curso de Paleontología General de la Escuela de Geología de la Universidad Industrial de Santander a la Formación Rosablanca en el municipio de Zapatoca 
(Santander, Colombia) durante el periodo 2012-2013, sobre la vía Zapatoca -cerro de La Vieja $\left(6^{\circ} 51^{\prime} 22,57 \mathrm{~N}\right.$ y $73^{\circ} 14^{\prime} 01,57$ O) (Figura 1).

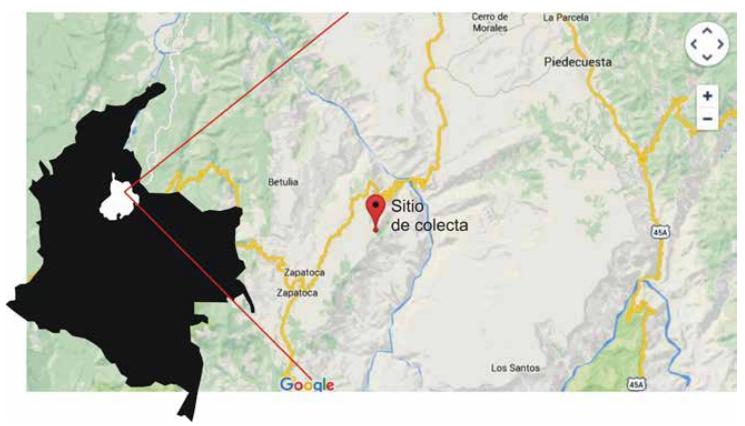

Figura 1. Lugar de recolección de los especímenes. Carretera al cerro de La Vieja (Zapatoca, Santander).

\subsection{Método de búsqueda de especímenes}

El método utilizado fue el de rastrillo, donde cada estudiante exploraba un área aproximada de $1 \mathrm{~m}^{2}$ durante tres minutos, hasta cubrir una línea de $10 \mathrm{~m}$. Los especímenes hallados fueron georreferenciados, etiquetados y embalados en bolsas ziploc. Para la descripción y determinación de los especímenes se utilizó la clave virtual para equinoideos del Museo de Historia Natural del Reino Unido (pág. web del Museo) además de la consulta de las descripciones de Cotteau et al. (1883, 1884), Kier (1972), Buitron (1978), Radwanska (1999) y la asesoría del doctor Andreas Kroh del Museo de Historia Natural de Viena.

La terminología básica para comprender la descripción del espécimen se presenta en este breve glosario:

ABORAL: parte del cuerpo opuesto a la boca.

ADAPICAL: hacia la parte más alta de la testa.

ADORAL: hacia la boca.

AMBULACROS: zona de las placas asociadas con el sistema vascular del agua y los pies-tubo. Generalmente biserial. Los erizos actuales presentan cinco zonas ambulacrales.

AMBITUS: punto más ancho en la circunferencia de la testa.

ÁREA INTERAMBULACRAL: pertinente a las placas que separan las zonas ambulacrales en la corona.

CORONA: placas del esqueleto calcítico excluyendo las placas peristomiales y apicales.

DISCO APICAL o SISTEMA APICAL: consta de un anillo de placas esqueletales especializadas que incluye las placas genitales y las placas oculares. Localizado en la parte más alta de la testa.

GONOPORO: una pequeña perforación a través de la placa genital que marca la apertura externa de las gónadas.

PERIPROCTO: área de la testa de los equinoideos donde se encuentra el ano.

PERISTOMA: área de la testa donde se encuentra la boca.

PLACA GENITAL: una de las placas del disco apical que yace interradialmente en la parte superior de un interambulacro y es traspasada por un gonoporo.

PLACA OCULAR: una de las placas del disco apical que yace radialmente en el ápice de una zona ambulacral. TESTA: la concha de un equinoideo.

2.1.1 Observaciones macro y microscópicas. Los caracteres de los especímenes fueron observados bajo el microscopio estereoscopio y con lupa de mano en el Laboratorio de Paleontología de la Universidad Industrial de Santander. Las fotografías se tomaron con una cámara Kodak de 12 Megapixeles

2.1.2 Análisis estadístico. La estadística descriptiva de la muestra así como las curvas de supervivencia y análisis de regresión se elaboraron utilizando el programa PAST 3.09. (Hammer et al. 2001)

2.1.3 Análisis sistemático. Para tratar de inferir las relaciones filogenéticas de la especie aquí descrita, se elaboró una matriz exploratoria binaria donde se consideró a $P$. depressa como grupo externo, debido a que es la especie más antigua (Bathoniano) del grupo de los Pseudocidaris analizados. De igual forma se consideraron nueve caracteres y las especies $P$. romani, $P$. thurmani, P. lusitánica, P.mammosa, P. sanctacrucensis y $P$. clunifera. El análisis se llevo a cabo en el programa winclada 1.00.08.

\section{RESULTADOS Y DISCUSIÓN}

El techo de la Formación Rosablanca en el área de estudio se caracteriza por presentar una fauna rica en bivalvos como Cucullae gabrielis, Trigonia sp, Inoceramus $s p$, gastrópodos como Ampulla sp, braquiópodos como Sellithyris sella, varias especies de ammonites, galerías tipo thallasinoides, equidermos como Toxaster colombianus, escudos de tortugas y dientes de reptiles entre otras especies. El paleoambiente fue marino de tipo somero con alta energía. 
Como parte del trabajo de campo en paleontología, que implica búsqueda y reconocimiento de especies,

se colectaron seis especímenes cuyas características se ajustan al género Pseudocidaris, que no aparece descrito para esta formación.

\subsection{Paleoecología}

Al igual que la mayoría de erizos regulares epifaunales de este tipo de ambientes, Pseudocidaris $s p$. debía alimentarse de algas que raspaba con su aparato masticador y de invertebrados, como serpúlidos, que tienen un buen registro fósil sobre las conchas.

La densidad baja de especímenes no permite determinar el tipo de distribución de esta especie. Los especímenes colectados muestran que la población presentaba una curva de supervivencia tipo II, lo que indica que las tasas de mortalidad variaban poco con la edad (Figura 2). Esto es extraño, ya que en los invertebrados es más común una tasa de mortalidad elevada en las primeras etapas de vida (curva de supervivencia tipo III), debido a la elevada producción de gametos liberados al medio acuático, como lo ejemplifica González-Peláez (2004) para el erizo actual Echinometra vanbrunti. Es muy probable que se esté enmascarando la muerte de estadios juveniles que debido a su tamaño no se conservaron o fosilizaron. El crecimiento de los individuos es isométrico $(\mathrm{y}=0,64 \mathrm{X}$ $-1,10$ t: 6,89 p: 0,$\left.002 ; r^{2}: 0,92\right)$ para las variables altura y diámetro.

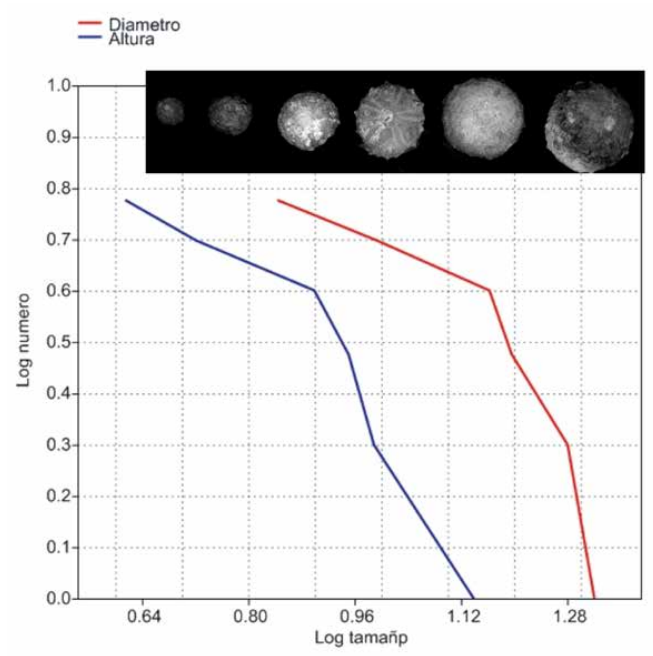

Figura 2. Curva de supervivencia de Pseudocidaris spp con base en las variables altura y diámetro.
La siguiente clasificación se basa en la propuesta de Kroh y Smith (2010).

Phylum Echinodermata

Clase Echinoidea

Subclase Euechinoidea

Superorden Carinacea Kroh y Smith (2010)

Orden Hemicidaroidea

Familia Hemicidaridae

Subfamilia Pseudocidarinae Smith y Wright (1993)

Genero Pseudocidaris Etallon 1859

Especie Pseudocidaris $s p$.

Diagnosis: ancho de la testa $(7-21 \mathrm{~mm})$, altura $(4,1-13,8$ $\mathrm{mm})$. La Tabla 1 resume los valores estadísticos de la muestra. La altura de la concha corresponde con un 57 $\%$ del diámetro (Figura 3). Testa baja y redondeada, disco apical dicíclico, periprocto grande pentagonal, placas oculares en forma de boomerang, gonoporos posicionados un poco más arriba del centro de las placas genitales. Ambulacros anchos desde la región apical, ampliándose un poco desde el ambitus, con tuberculaciones unigeminadas hasta la zona adoral, y trigeminadas en la región oral con tubérculos grandes, pero más pequeños que los interambulacrales. Área interambulacral compuesta de 4 a 5 placas con un tubérculo primario crenulado y perforado, areolas con 14 tubérculos escrobiculares. En el ápice, una columna presenta un tubérculo interambulacral primario, mientras que la placa adapical es más pequeña y con tubérculo pequeño (Figura 4).

Tabla 1. Estadística univariada de los especímenes de Pseudocidaris spp.

\begin{tabular}{lrr}
\hline & \multicolumn{1}{l}{ Diámetro } & \multicolumn{1}{c}{ Altura } \\
$\mathrm{N}$ & 6 & 6 \\
Min & 6.96 & 4.11 \\
Máx & 20.89 & 13.77 \\
Media & 14.485 & 8.281667 \\
Std. Error & 2.170341 & 1.40577 \\
Variance & 28.26227 & 11.85714 \\
Stand. Dev & 5.316227 & 3.443419 \\
Skewness & -0.3353978 & 0.5023287 \\
Kurtosis & -1.181372 & 0.2132898 \\
\hline
\end{tabular}

La especie de Pseudocidaris de Zapatoca se diferencia de $P$. mammosa, P. thurmanni, $P$. sanctacrucencis, $P$. clunifera y $P$. lusitánica, en que presenta los ambulacros anchos y poco sinuosos y el disco apical dicíclico. 
Debido a la poca sinuosidad de los ambulacros, el Pseudocidaris de Zapatoca puede estar emparentado con las especies jurásicas $P$. depressa (Bathoniano), pero se diferencia en la estructura del disco apical, ya que esta última presenta la tercera placa genital separada del periprocto y $P$. alhadacensis (Calloviano), que presenta la testa elevada y los ambulacros delgados en la región apical.

Esta hipótesis inicial fue evaluada mediante el análisis filogenético que produjo un único árbol (Figura 5), el cual mostró que la especie de Zapatoca está relacionada con $P$. lusitánica y P. clunifera. El grupo hermano de este clado es $P$. romani, cuya relación está definida por el disco apical dicíclico. Los índices de consistencia (IC:72) y retención (IR: 70) indican un soporte relativo del árbol. La hipótesis filogenética obtenida muestra que el linaje de la especie en cuestión se origina en el Calloviano (Jurásico) y no, como se pensaba, en el Bathoniano con P. depressa.

Los caracteres testa baja, disco apical dicíclico, ambulacros anchos poco sinuosos, periprocto pentagonal, pueden sugerir que el Pseudocidaris de Zapatoca sea una nueva especie.

Es presumible que esta especie haya sido descrita en el pasado como una sinonimia de Pseudocidaris, debido a que no se encontró registro de este género para la Formación Rosablanca. El único registro del género lo realizó Patarroyo (2002) para el Cretácico inferior de Firavitoba (Boyacá).

Estudios filogenéticos más profundos deben realizarse para determinar la novedad de la especie.

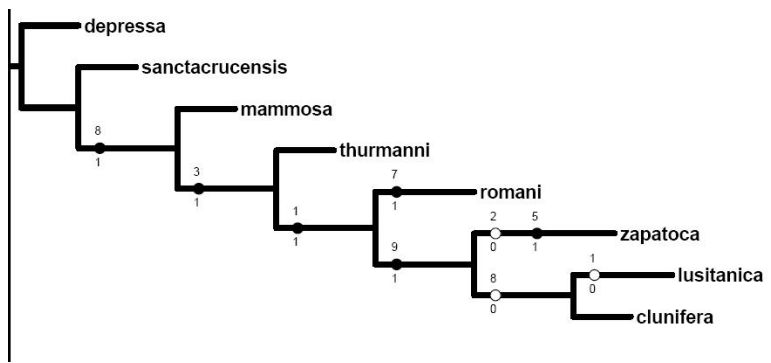

Figura 5. Árbol filogenético de Pseudocidaris. La especie aquí descrita esta rotulada como "Zapatoca", siendo el grupo hermano de $P$. lusitánica y P. clunifera.

\section{REFERENCIAS}

Buitron, B. E. (1978). Hemicidaridos (Echinodermata, Echinoidea) del Jurásico Superior de Oaxaca y Chiapas. Univ. Auton. Mexico, Inst. Geología. Revista 2, 65-68.

Cotteau, M.M., Peron \& Gauthier (1883). Echinides Fossiles de L'Algerie. Premier Fascicule. Paris: G. Masson.

Cotteau, M.M., Peron \& Gauthier. (1884). Echinides Fossiles de L'Algerie. Deuxieme Fascicule. Paris: G. Masson.

Etayo-Serna, F. (1964). Posición de las faunas en los depósitos Cretácicos colombianos y su valor en la subdivisión cronológica de los mismos. Boletín de Geología, 16 (17), 141.

Glossary of Terms of Echinoderms. Southeastern Regional Taxonomic Center South Carolina Department of Natural Resources. Retrieved from http://www.dnr.sc.gov/marine/sertc/.

González-Peláez, S.S. (2004). Biología poblacional del erizo Echinometra vanbrunti (Echinodermata:Echinoidea) en el sur del Golfo de California, México. Tesis de Maestría. Centro de Investigaciones Biólogicas del Noroeste, SC.

Guzmán, G. (1985). Los Griferidos Infracretácicos.

Aetostreon couloni y Ceratostreon boussingaulti,de la Formación Rosablanca, como indicadores de oscilaciones marinas. Proyecto Cretácico. Bogotá: Publicaciones Geológicas Especiales del Ingeominas.

Hammer, Ø., Harper, D.A.T. \& Ryan, P.D. (2001). PAST: Paleontological statistics software package for education and data analysis. Palaeontologia Electronica, 4(1), 9. Retrieved from http://palaeo-electronica.org/2001_1/past/issue1_01. htm.

Kier, M. P. (1972). Tertiary and Mesozoic Echinoids of Saudi Arabia. Smithsonian Contributions to Paleobiology. (10). 
Kroh, A., \& Smith, A.B. (2010). The Phylogeny and Classification of post-Paleozoic echinoids. Journal of Systematic Palaeontology, 8(2), 147-212. doi:10.1080/14772011003603556.

Patarroyo, P. (2002). Equinoideos del miembro calcáreo superior, Formación Tibasosa, en el área de Firavitoba (Boyaca, Colombia), Morfología y Fauna asociada. Geología Colombiana, 27, 95107. doi: $10.15446 / g c$.

Radwanska, U. (1999). Lower Kimmeridgian Echinoids of Poland. Acta Geologica Polonica, 49(4), 287364.

Smith, A. B. \& Kroh, A. (ed.) (2011). The Echinoid Directory. World Wide Web electronic publication. Retrieved from http://www.nhm.ac.uk/ research-curation/projects/echinoid-directory.

Ward, D., Goldsmith, R.., Cruz, B., Jaramillo, C. \& Restrepo, H. (1973). Geología de los Cuadrángulos H-12, Bucaramanga y H-13, Pamplona, departamento de Santander. U.S. Geological Survey e INGEOMINAS. Boletín Geológico, XXI (1-3), 1-132.
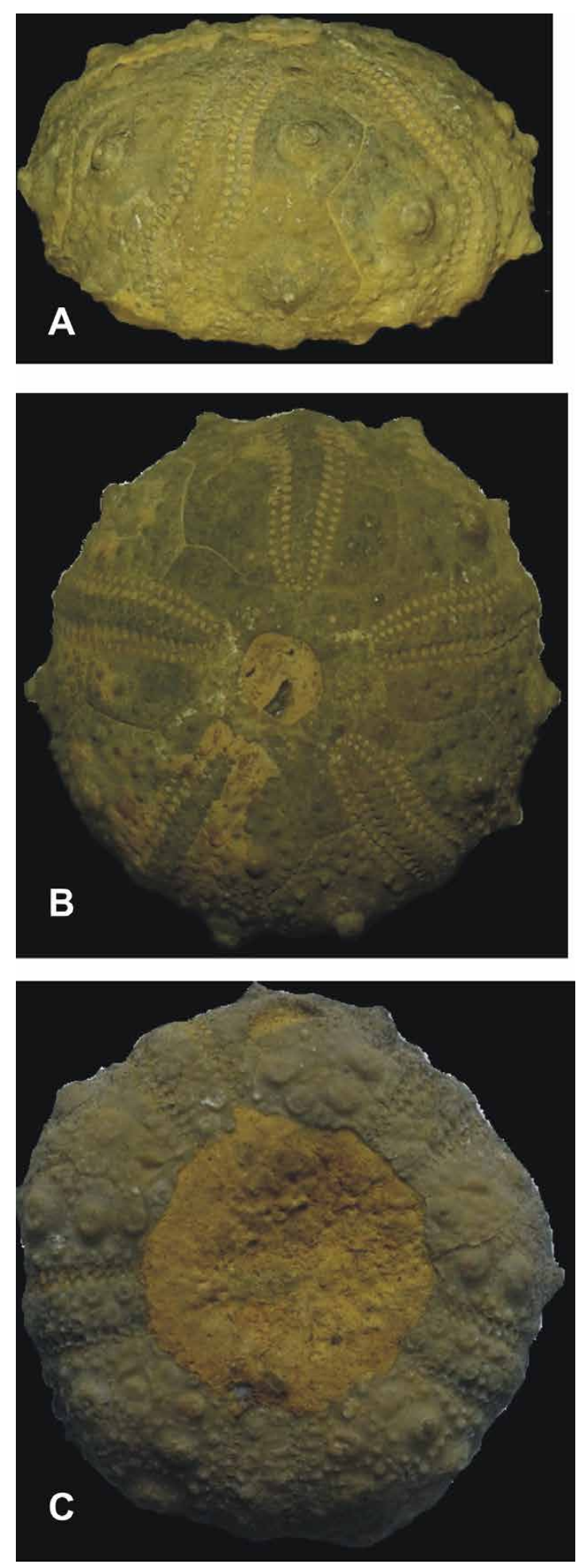

Figura 3. Pseudocidaris sp. A. Vista lateral. Ambitus y ambulacro. B. Vista aboral. C. Vista oral. 
Nuevo registro de pseudocidaris spp. (Echinoidea: hemicidaridae) del cretácico inferior (Valanginiano-hauteriviano) de Zapatoca (Santander, Colombia)
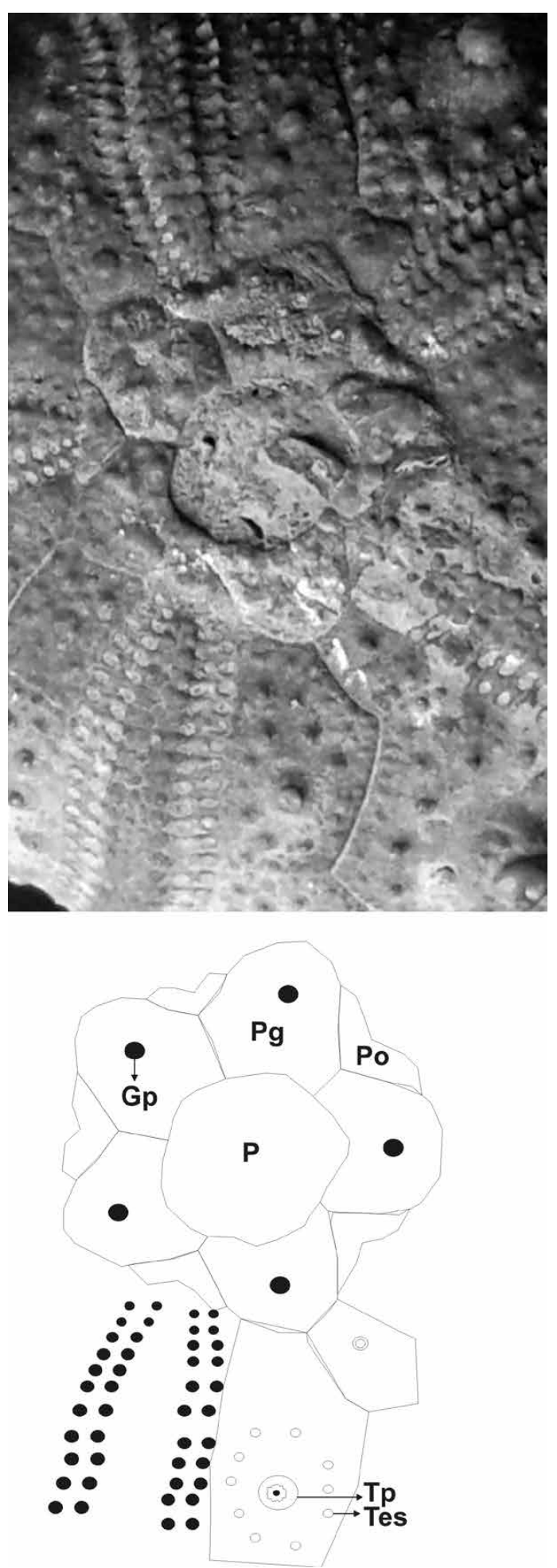

Figura 4. Pseudocidaris sp. Disco apical. Gp: gonoporo, P: periprocto. Pg: Placa genital. Po: Placa ocelar. Tes: tubérculo escrobicular. Tp: tubérculo primario. 